\title{
Atomic configurations in AP-MOVPE grown lattice-mismatched InGaAsN films unravelled by X-ray photoelectron spectroscopy combined with bulk and surface characterization techniques
}

\author{
M.C. López-Escalante , B. Ściana , W. Dawidowski , K. Bielak , M. Gabás
}

\begin{abstract}
A B S T R A C T
This work presents the results of X-ray photoelectron spectroscopy studies on the bonding $\mathrm{N}$ configuration in InGaAsN epilayers grown by atmospheric pressure metal organic vapour phase epitaxy. Growth temperature has been tuned in order to obtain both, relaxed and strained layers. The studies were concentrated on analysing the influence of the growth temperature, post growth thermal annealing process and surface quality on the formation of Ga-N and In-N bonds as well as N-related defects. The contamination of InGaAsN films by growth precursor residues and oxides has also been addressed. The growth temperature stands out as a decisive factor boosting In- $\mathrm{N}$ bonds formation, while the thermal annealing seems to affect the $\mathrm{N}$-related defects density in the layers.
\end{abstract}

\section{Introduction}

GaAs and InGaAs dilute nitrides, into which small concentrations of nitrogen have been alloyed (GaAsN and InGaAsN, respectively), have been extensively studied from the end of the nineties of the 20th century [1,2]. These semiconductor alloys exhibit unique properties, such as the composition dependent negative and huge bowing coefficient (up to $40 \mathrm{eV}$ ) [3], and the large conduction band offset ( $>300 \mathrm{meV}$ ) [2]. They are a consequence of the larger electronegativity of $\mathrm{N}$ (3.04), as compared to As (2.18), and also, of the big difference between As and $\mathrm{N}$ atomic radii, 115 and $65 \mathrm{pm}$, respectively. The cohesive energies of the respective binary compounds creating the InGaAsN quaternary alloys is as follows: $\mathrm{GaN}>\mathrm{InN}>\mathrm{GaAs}>\operatorname{InAs}(2.24,1.93,1.63$, and $1.55 \mathrm{eV}$ per bond, respectively) [4]. Therefore, during the epitaxial growth of these compounds, the atomic configurations driving force will be the maximization of the cohesive bond energy (which leads to the formation of Ga-N and In-As bonds), instead of the minimization of local strains (which would favour the formation of In-N and Ga-As bonds).
Partial substitution of arsenic with nitrogen atoms in GaAs host material induces a lattice constant reduction and, as a consequence, it strongly modifies the electronic structure (band gap reduction, conduction band splitting), and the layer optical properties (drastic deterioration of photoluminescence, absorption redshift) $[5,6]$. The introduction of indium into GaAsN alloy leads to a further band gap decrease and compensates stresses induced by nitrogen atoms. For certain compositions, (three times more indium than nitrogen), quaternary InGaAsN alloys can be grown lattice-matched to GaAs and Ge substrates. Diluted nitrides are very promising for applications in telecom-wavelength GaAs-based emitters (LEDs and VCSEL) $[7,8]$ and in very efficient multijunction solar cells [9].

However, the epitaxial growth of a high quality material InGaAsN alloy is very difficult due to a large miscibility gap between arsenides and nitrides. They exhibit a metastable nature and require low growth temperatures. This is why the main epitaxial methods recommended for dilute nitrides are low pressure metalorganic vapour phase epitaxy (LP-MOVPE) and molecular beam epitaxy (MBE). Higher growth temperatures improve optical quality of InGaAsN but promote the phase segregation and composition inhomogeneity. Up to now, main efforts of the growers have been focussed on understanding the reasons and nature of defects generated in InGaAsN alloys, and on the optimisation of the growth parameters and post growth annealing. This purpose requires the 
application of a wide spectrum of characterisation methods such as high resolution X-ray diffraction (HRXRD), secondary ions mass spectrometry (SIMS), Raman spectroscopy (RS), photoluminiscence (PL), scanning and transmission electron microscopies (SEM, TEM), atomic force microscopy (AFM), deep level transient spectroscopy (DLTS), X-ray photoelectron spectroscopy (XPS), and many others.

Nearest-neighbour bonding configuration of the isovalent nitrogen atom, i.e. the ratio between Ga-N and In-N bonds, has the major influence on the electronic properties and lattice strains in InGaAsN alloys. A higher amount of In-N bonds decreases the structural strains (large cation-small anion bond) and is responsible for the blueshift of PL spectra. Moreover, the crystalline quality of InGaAsN films suffers from a large amount of point defects (vacancies, antisites, interstitials) and a high level of impurities incorporated especially during MOVPE growth (oxygen, carbon, hydrogen) [10]. The most probable defects formed in InGaAsN alloys to minimize the local strains are $\mathrm{N}$ complexes such as $(\mathrm{N}-\mathrm{N})_{\mathrm{i}}$ and $(\mathrm{N}-\mathrm{As})_{\mathrm{i}}$, split interstitials and $\mathrm{N}_{\mathrm{As}}-\mathrm{As}_{\mathrm{Ga}}$ nearest neighbour pairs [4,11]. Therefore, the knowledge of the factors ruling the $\mathrm{N}$ coordination chemistry in these layers is fundamental for achieving the best growth conditions.

XPS is one of the most convenient method for the determination of the N-bonding configuration in as grown InGaAsN epilayers and its changes after post growth annealing (rapid thermal annealing process - RTA) [12-14].

This work presents the results of XPS studies of the changes induced by $\mathrm{N}$-bonding configuration in InGaAsN epilayers grown by atmospheric pressure (AP)-MOVPE. XPS analysis of InGaAsN films grown by this epitaxial method is a very difficult task due to the high probability of incorporation of different impurities connected with oxygen and precursor residues on the layer surface. Additionally, the investigated structures reveal different level of strain relaxations what influences on surface morphology and chemistry. Therefore, the presented XPS investigation is supported by other characterisation techniques such as HRXRD (composition changes and strain analysis), SEM and AFM (impact of thermal annealing on surface morphology).

\section{Experimental}

The investigated structures were grown by AP-MOVPE using AIX 200 R\&D horizontal reactor on (100)-oriented silicon doped $n$-GaAs:Si substrates. Trimethylgallium TMGa $\left(\mathrm{Ga}\left(\mathrm{CH}_{3}\right)_{3}\right)$, trimethylindium TMIn $\left(\operatorname{In}\left(\mathrm{CH}_{3}\right)_{3}\right)$, tertiarybutylhydrazine TBHy $\left(\left(\mathrm{C}_{4} \mathrm{H}_{9}\right) \mathrm{HN}_{2} \mathrm{H}_{2}\right)$ and arsine $\mathrm{AsH}_{3}\left(10 \%\right.$ of $\mathrm{AsH}_{3}$ in $\left.\mathrm{H}_{2}\right)$ were used as growth precursors. The epitaxial structures consisted of two undoped layers: $0.4 \mu \mathrm{m}$ thick GaAs buffer grown at $670^{\circ} \mathrm{C}$ and InGaAsN layer deposited at $585^{\circ} \mathrm{C}$ during $10 \mathrm{~min}$ (sample NI74n) and at $575^{\circ} \mathrm{C}$ during $30 \mathrm{~min}$ (sample NI52n). Different growth times were set in order to obtain strained and relaxed InGaAsN layers. For the GaAs buffer growth, the flow rates were $19.6 \mu \mathrm{mol} / \mathrm{min}$ (TMGa) and $1339.3 \mu \mathrm{mol} / \mathrm{min}\left(\mathrm{AsH}_{3}\right)$, giving thus a $\mathrm{V} / \mathrm{III}$ ratio of 68.3, while for InGaAsN layer growth, flow rates of 3.0, 13.7, 223.2 and $445.6 \mu \mathrm{mol} / \mathrm{min}$ were set for TMIn, TMGa, $\mathrm{AsH}_{3}$ and TBHy, respectively (V/III ratio 40.1). Detailed information about growth process can be found elsewhere $[15,16]$. As it is well known, the post growth thermal annealing induces atomic configuration rearrangements and removes some structural defects what should improve the optical properties of the dilute nitride structures [17]. In order to investigate the potential impact of thermal process on nitrogen and indium bonding configuration in InGaAsN epilayers a rapid thermal annealing (RTA) was applied under $\mathrm{N}_{2}$ flow at $700^{\circ} \mathrm{C}$ during $5 \mathrm{~min}$ to both as-grown samples. To prevent the evaporation of V-group elements, a clean GaAs wafer was placed face to face on the sample surfaces during RTA treatment. Therefore, four samples have been studied in this work, two as-grown samples, NI52n and NI74n, and the samples prepared in the same conditions but after a RTA, NI52 nA and NI74nA.

The characterisation of the obtained structures was made by means of HRXRD, PL, SEM and AFM. The structural properties of InGaAsN layers (composition, thickness and strain relaxation) were determined by measurements of diffraction rocking curves and reciprocal space maps (RLMs) using Philips MRD High Resolution X-Ray Diffractometer. The system has a four-crystal Bartels (2 20 ) monochromator giving a well-collimated (12 arcs divergence) beam of CuK $\alpha 1$ radiation ( $1.540597 \AA$ ). The diffraction rocking curves were obtained by scanning in the $\omega / 2 \theta$ geometry about the GaAs (004) symmetric reflection. The RLMs were recorded for (224) asymmetrical reflection in order to reveal the strain relaxation in different crystallographic directions. The optical properties were analysed based on photoluminescence spectra recorded using a single-grating $0.3 \mathrm{~m}$ focal length monochromator with an InGaAs CCD camera as a detecting source. A semiconductor laser $(639 \mathrm{~nm}$ and $10 \mathrm{~mW}$ excitation power) was used as the excitation source. Samples were mounted on a cold finger in a helium closed-cycle refrigerator which allows to carry out measurements in low temperature $(15 \mathrm{~K})$. Planar view images of the sample surfaces were taken with a FEI Helios Nanolab 650 Dual Beam microscope, fitted with a Schottky field emission source for SEM (FESEM) and a Tomahawk focused ion beam. AFM images were made in air, in tapping mode with application of standard silicon probes with $\sim 10 \mathrm{~nm}$ nominal tip radius using Bruker Multimode V AFM setup. Scanning speed was set to $5 \mu \mathrm{m} / \mathrm{s}$. The image processing was performed using Open Source Gwyddion software.

The In and $\mathrm{N}$ coordination chemistry was investigated through XPS.XPS spectra were obtained using an X-ray photoelectron spectrometer (Physical Electronics, Inc., model PHI 5700) with a $300 \mathrm{~W}$, $\mathrm{Mg} \mathrm{K} \alpha(1253.6 \mathrm{eV})$ excitation source, at a fixed voltage of $15 \mathrm{kV}$ and the vacuum pressure reaches $10^{-9}$ Torr in the XPS analysis chamber. The spatial resolution and the spot size are defined by the analyzer aperture, which is $720 \mu \mathrm{m}$ in diameter. Angle resolved XPS (ARXPS) data were obtained at several angles of emission. Core level deconvolution in the several peak components has been made using the XPSPeak software package [18]. Shirley background subtractions have been applied to each analysed core level and the peak line shapes are Gaussian-Lorentzian (70-30).

\section{Results and discussion}

Thickness and composition inhomogeneity, which comes up spontaneously during the growth of InGaAsN epilayers, were determined from the simulations of the measured diffraction rocking curves for (004) reflection (Fig. 1), taking into account the bandgap value evaluated from contactless electroreflectance (CER) spectra. Detailed information about employed algorithm was published previously $[19,20]$. The data analysis reveals both, indium and nitrogen content gradients, no matter growth temperature nor the RTA treatment. This fact can be explained on the basis of the dominance of surface kinetics, nonlinear dependence of $\mathrm{N}$ incorporation on In content, and also of the strain gradient effect on the effective diffusion of $\mathrm{N}$ [21]. In the case of the sample NI52n (Fig. 2a), the simulations indicated a composition gradation. In content increases toward the sample surface from $3.8 \%$ to $7 \%$ through the first $172 \mathrm{~nm}$, then from $7 \%$ to $11 \%$ through $6 \mathrm{~nm}$ and finally from $11 \%$ to $20.5 \%$ through $32 \mathrm{~nm}$ while $\mathrm{N}$ content decreases toward the sample surface from $0.68 \%$ to $0.48 \%$ through the first $172 \mathrm{~nm}$ and remains constant of $0.48 \%$ through the next $38 \mathrm{~nm}$. The total thickness of the deposited InGaAsN layer is $210 \mathrm{~nm}$. For the sample NI74n (Fig. 2b), a similar tendency has been found, In content increases toward the sample surface from $8.3 \%$ to $12.2 \%$ through the first $65 \mathrm{~nm}$, then 
a)

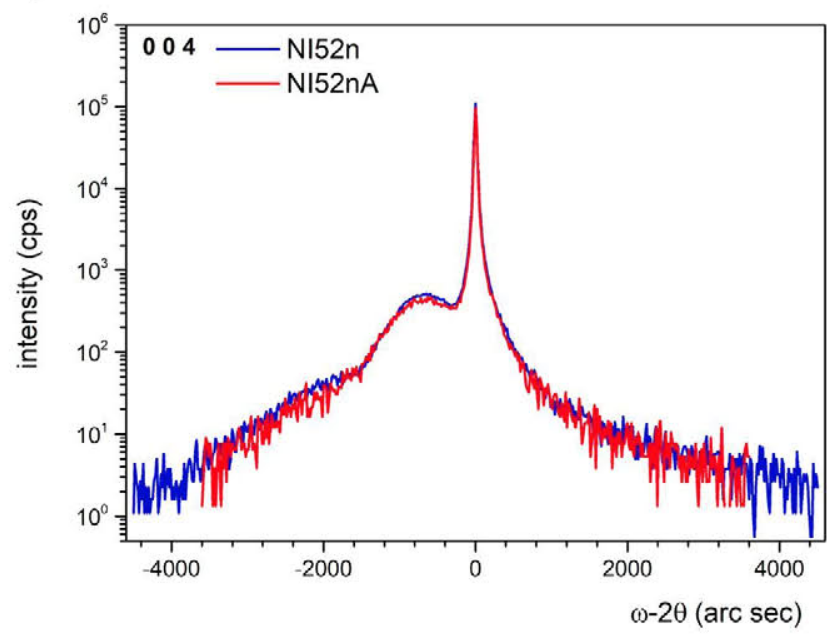

b)

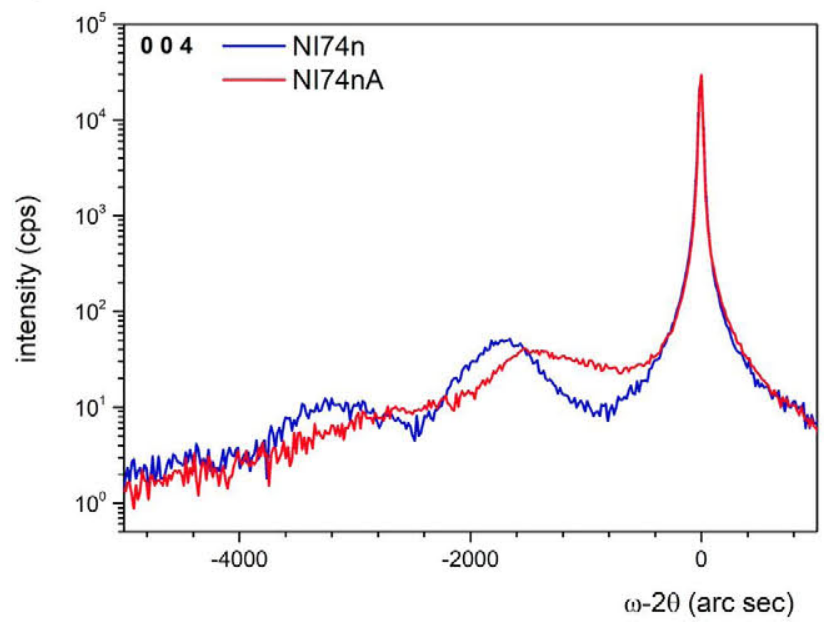

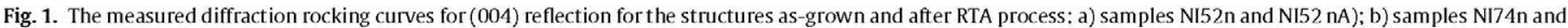
NI74 nA.

\begin{tabular}{|c|c|c|}
\hline \multicolumn{3}{|c|}{ NI52n (NI52nA) } \\
\hline \multicolumn{3}{|c|}{$20.5 \%$} \\
\hline $\mathrm{i}-\operatorname{InGaAsN}: \operatorname{In}{ }_{11 \%}$ & $\mathrm{~N}=0.48 \%$ & $-32 n m$ \\
\hline 119 & & \\
\hline$i-\operatorname{InGaAsN}: \ln _{7 \%}$ & $\mathrm{~N}=0.48 \%$ & $-6 n m$ \\
\hline & $0.48 \%$ & \\
\hline $\mathrm{i}-\operatorname{InGaAsN}: \ln { }_{3.8 \%}$ & $N \backslash$ & $-172 n m$ \\
\hline i-GaAs "buffer" & oped & $-400 n m$ \\
\hline
\end{tabular}

b) $\quad \mathrm{N} 174 \mathrm{n}$

\begin{tabular}{|ll|}
\hline i-InGaAsN: In $\left.\right|_{13 \%} ^{24 \%} \mathrm{~N}=0.2 \%$ & $\sim 20 \mathrm{~nm}$ \\
\hline i-InGaAsN: In $\left.\right|_{8.3 \%} ^{12.2 \%} 0.3 \%$ & $-65 \mathrm{~nm}$ \\
\hline i-GaAs "buffer" undoped & $\sim 400 \mathrm{~nm}$ \\
\hline substrate GaAs (n-type) & \\
\hline
\end{tabular}

c)

NI74nA

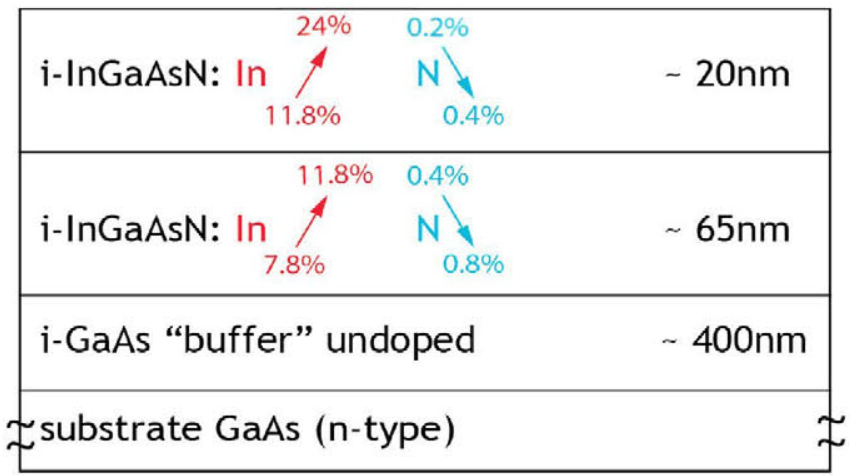

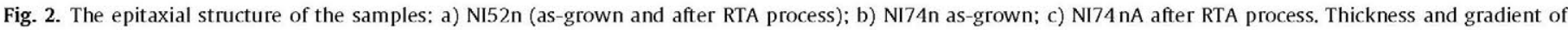
composition of InGaAsN epilayers were determined from the simulation of the measured diffraction rocking curves.

from $13 \%$ to $24 \%$ through the final $20 \mathrm{~nm}$. On the contrary, $\mathrm{N}$ content decreases toward the sample surface from $0.5 \%$ to $0.3 \%$ through the first $65 \mathrm{~nm}$ and remains constant, $0.2 \%$, through the final $20 \mathrm{~nm}$, so the total thickness of this InGaAsN layer is $85 \mathrm{~nm}$. These figures allow to deduce that the higher growth temperature, the more difficult $\mathrm{N}$ incorporation into the InGaAs lattice.
To check the influence of RTA process on the structural properties of InGaAsN epilayers the diffraction rocking curves and reciprocal space maps (RSMs) of the samples NI52 nA and NI74 nA (after RTA treatment) were measured. No changes in structural characteristics, nor in the layer composition gradation have been detected for the sample NI52 nA (red curve in Fig. 1a), as compared 
a)

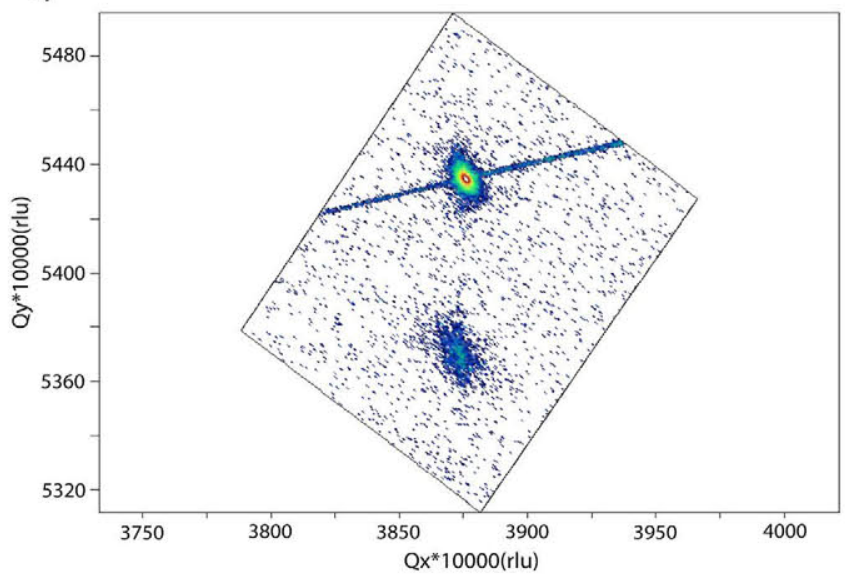

b)

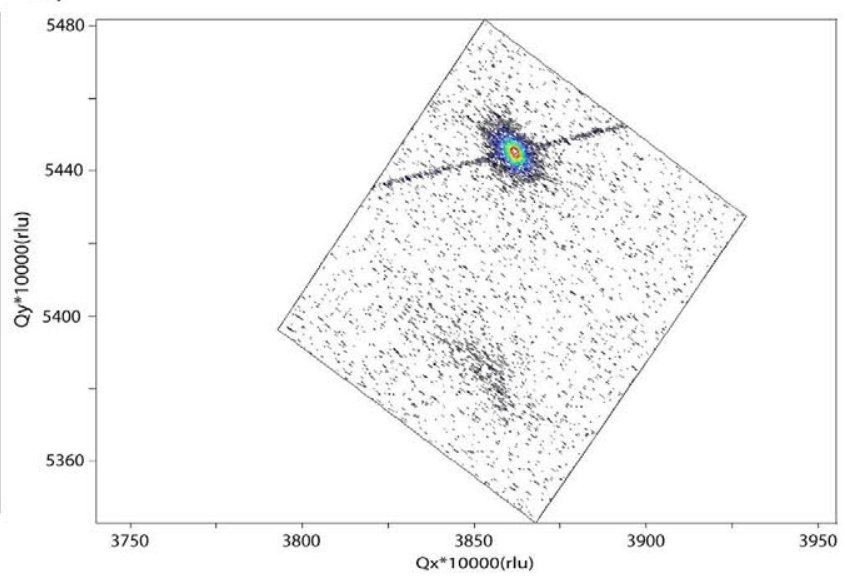

Fig. 3. The reciprocal space maps for the (224) asymmetrical reflection of the samples: a) NI74n as-grown; b) NI74 nA after RTA process.

to NI52n (blue curve in Fig. 1a), which is probably connected with the observed relaxation of this layer before RTA process. The immediate consequence is that there is no $\mathrm{N}$ incorporation in this layer after RTA. Unlike to the sample NI52 nA, several changes of the structural parameters are noticeable after the annealing of the sample NI74n (Fig. 1b and Fig. 3b). The rocking curve corresponding to the sample NI74nA (red curve in Fig. 1b) is blurry, the reflection connected with InGaAsN epilayer has a low intensity and shifts toward the GaAs substrate reflex. This behaviour is connected with an increase of both the sample relaxation and the nitrogen content. Additionally, the simulation of the (004) rocking curve reflection of NI74 nA sample revealed the composition changes showing an increase of nitrogen content. The thickness and composition gradient in each NI74 nA sublayer are presented in Fig. 2c. The indium content increases from $7.8 \%$ (8.3\% before RTA) to $24 \%$, while nitrogen amount decreases from $0.8 \%$ ( $0.5 \%$ before RTA) to $0.2 \%$ (for NI74n) toward the sample surface. In summary, $\mathrm{N}$ and In content in the InGaAsN layer keep same values after RTA for the sample prepared at low temperature. On the contrary, there is a small In amount diminution, accompanied by an effective $\mathrm{N}$ incorporation after RTA for the sample prepared at higher temperature.

The very small quantity of nitrogen in comparison to indium concentration generates compressive strains inside the InGaAsN epilayers what may cause the lattice relaxation. The value of the lattice relaxation evaluated from the reciprocal space maps of the (224) asymmetrical reflection is significant (about 97.5\%) for the sample NI52n (not shown), and much lower (about 7.9\%) for the sample NI74n (Fig. 3a). This is connected with the bigger thickness of the sample NI52n $(210 \mathrm{~nm})$, in comparison to that of the sample NI74n ( $85 \mathrm{~nm}$ ), and may be to the smaller $\mathrm{N}$ amount in the NI74n sample (Fig. 2b), which will induce a higher compressive strain in this lattice. According to the absence of changes in the (004) reflection for the NI52 nA sample, the (224) reflection RSMs corresponding to NI52n and NI52 nA samples do not show any difference between them. On the contrary, RTA process increases the relaxation in the NI74 nA layer, whose estimated value is now $30 \%$, as evaluated from the measured reciprocal space map (Fig. 3b). This relaxation rising is accompanied by a noticeable increase in the N amount observed in the NI74 nA layer as compared to NI74n (Fig. 2b, c), though the value at the topmost surface is the same (0.2\%).

The lattice relaxation increases the surface roughness because of the generation of misfit dislocations (MDs) at the interface InGaAsN/GaAs, oriented along two orthogonal $<110\rangle$ crystallographic directions. They are distinctly visible in the AFM image of the almost fully relaxed sample NI52n (Fig. 4a), which shows the typical crosshatch morphology surface. No traces of this morphology can be seen on the NI74n surface, since the almost total absence of lattice relaxation inhibits the formation of MDs. However, a high density of small clear spots is detected on top of this surface. They are probably connected with precursor debris, whose more important presence in the sample deposited at high temperature will be confirmed in XPS measurements. These precursor debris would be responsible for the higher RMS of the NI74n sample, compared to NI52n.

Since RTA treatment does not change the relaxation degree of the crystalline lattice for the NI52 nA sample, the observed RMS increase for this sample could be a consequence of the As evaporation during RTA, in spite of the GaAs proximity cap used to mitigate this undesirable effect, which gives rise to the small voids visible on the sample surface. After the RTA treatment, NI74 nA saple exhibits a smaller RMS value, $1.26 \mathrm{~nm}$ compared to $1.49 \mathrm{~nm}$, in spite of the increased lattice relaxation which would promote the formation of MDs, and of the foreseeable As evaporation, as for the NI52 nA sample. However, there are no bright spots on this sample surface, which would be indicative of its evaporation during RTA treatment, and this could be the reason for the RMS diminution.

The inspection of the sample surfaces through SEM micrographs corroborates the AFM findings. Fig. 5 shows tilted pictures corresponding to as grown (left) and after RTA (right) samples. For the sample prepared at lower temperature, NI52n, elongated bulges can be observed on layer surface. For the NI74n layer (Fig. 5 bottom), these bulges look much smaller, which suggests the dislocations appearing after layer relaxation could be the origin of such features. On the other hand, the density of small white spheres is higher for the NI74n sample, which suggest a more important presence of precursor debris, as it was point out by AFM images.

NI52 nA sample surface (Fig. 5 top right) does not show noticeable differences when compared to NI52n, except for the appearance of some void due to As evaporation during RTA, as already seen in AFM images. These voids are also visible in NI74 nA surface, and its number seems to be a bit higher than in the NI52 nA sample surface. Regarding the presence of precursor debris, density of white spheres is smaller after RTA, especially for the NI74 nA sample. It is therefore probable that the bulges seen on NI52n and NI52 nA could act as an anchorage for the precursor debris, hindering thus its evaporation during RTA.

PL spectra were recorded at $15 \mathrm{~K}$ (laser diode operating at $639 \mathrm{~nm}$ with $10 \mathrm{~mW}$ optical power) for all investigated samples, as grown and after RTA process, and are presented in Fig. 6 . For the couple of samples NI74n and NI74 nA (Fig. 6b), a clear peak corresponding to InGaAsN layer is visible at $\sim 1.33 \mathrm{eV}$, accompanied 
a)

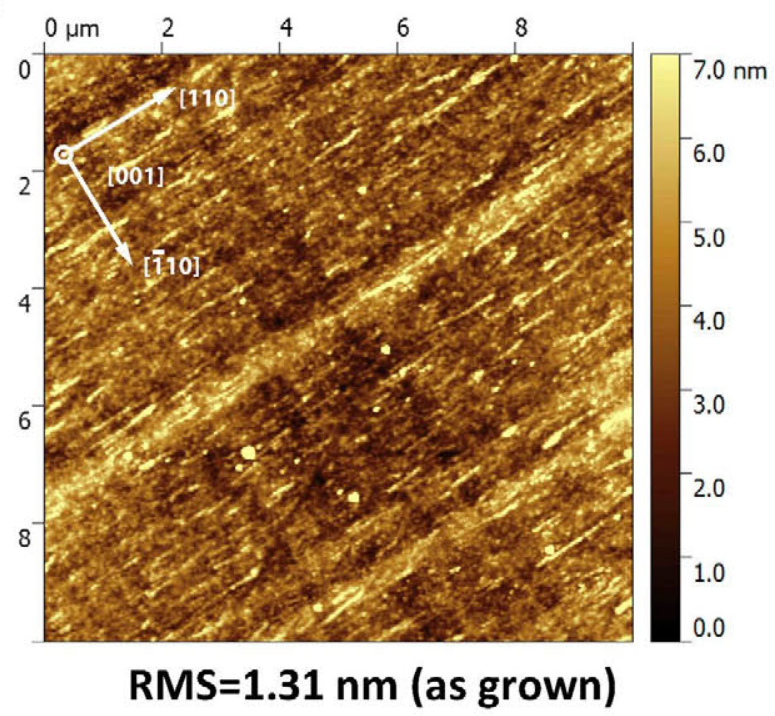

b)

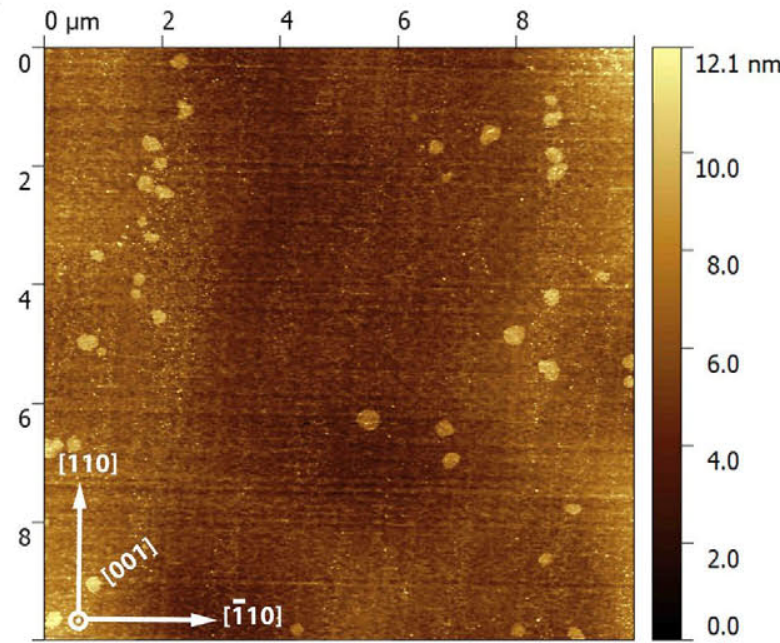

RMS=1.49 nm (as grown)

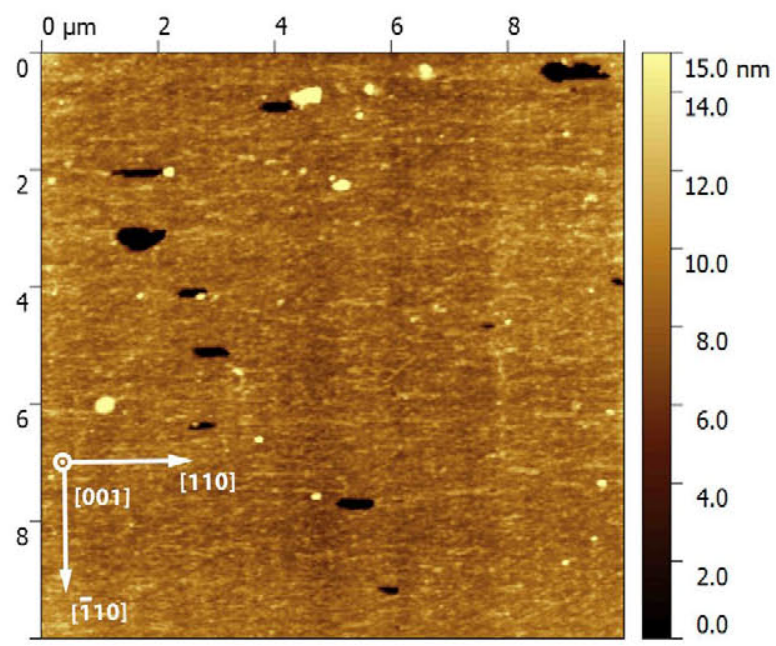

RMS=2.73 nm (after RTA)

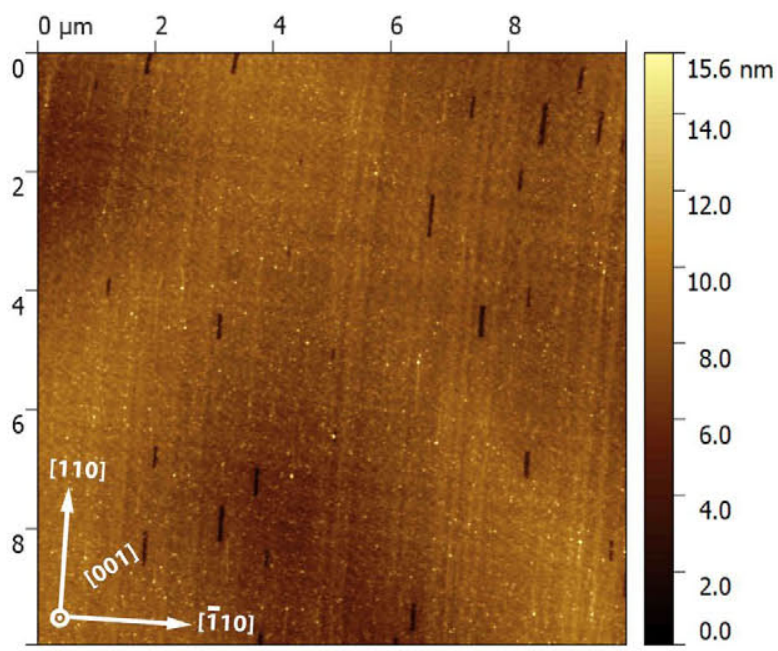

RMS=1.26 nm (after RTA)

Fig. 4. AFM images of investigated samples: a) NI52n as grown (left) and after RTA process (right); b) NI74n as grown (left) and after RTA process (right).

by the defect responses to excitation in the low energy region, on the left side of InGaAsN signal. A little redshift $(\sim 12 \mathrm{meV})$ of the main peak can be measured after RTA process (red line). It can be connected with the formation of new Ga-N bonds because of the As evaporation induced by the annealing process. This hypothesis would be supported by the XPS analysis of the Ga2 $\mathrm{p}_{3 / 2}$ core level (see SI file). Additionally, the lower intensity and broadening of this signal could be connected to an increasing presence of some N-related defects (N-N or N-As) and/or to the higher lattice relaxation after RTA (Fig. 3b). Moreover, the enhancement of the defect related emission band near $1 \mathrm{eV}$ would confirm the higher $\mathrm{N}-$ related defects density in the NI74 nA sample. In the case of almost fully relaxed samples NI52n and NI52nA, just the response from defects can be detected. A very broad emission band connected with N-related defects is usually observed in low temperature PL spectra of dilute nitrides, especially with small amount of nitrogen. It is due to the presence of a large amount of non-homogenous distributed nitrogen clusters leading to an increase of the conduction band tail states $[22,23]$. In the case of the sample NI52n, grown at lower temperature, the density of these defects is higher than for the sample NI74n and therefore the optical transitions connected with N-related states are the main features in this spectrum. RTA process boosts the formation of some type of defects (those associated to bands at $\sim 0.88 \mathrm{eV}$ and at $0.9-1 \mathrm{eV}$ as for the sample NI74 $\mathrm{nA}$ ) while some others diminish their density $(\sim 1.1 \mathrm{eV})$. The weak signal at about $1.3 \mathrm{eV}$ comes probably from the bandgap transition of the relaxed InGaAsN layer.

\subsection{XPS analysis}

A detailed analysis of the surface chemistry has been performed for the four samples at three different explored depths. The $\mathrm{C} 1 \mathrm{~s}$, $01 \mathrm{~s}, \mathrm{Ga} 2 \mathrm{p}_{3 / 2}, \mathrm{As} 2 \mathrm{p}_{3 / 2}$, As3d, In $3 \mathrm{~d}$ and N1 s core levels were measured. Only results from In $3 \mathrm{~d}$ and $\mathrm{N} 1 \mathrm{~s}$ core levels are going to be shown in this section, the details concerning the rest of core levels being part of the Supplementary Information (SI) file.

\subsubsection{N1s core level}

This core level can be deconvoluted in four different contributions, no matter sample or explored depth, as it is shown in Fig. 7a for the NI52n sample. The explored depth indicated in the figure is an estimation of the photoelectrons maximum length escape. 
a)
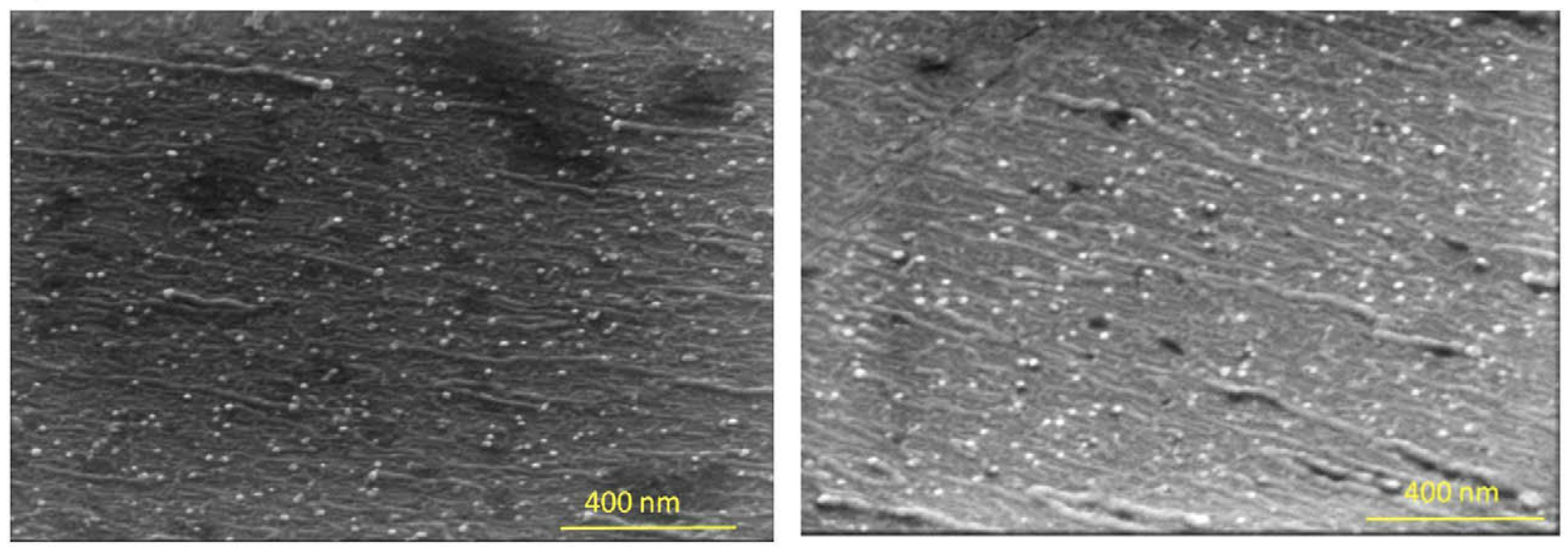

b)
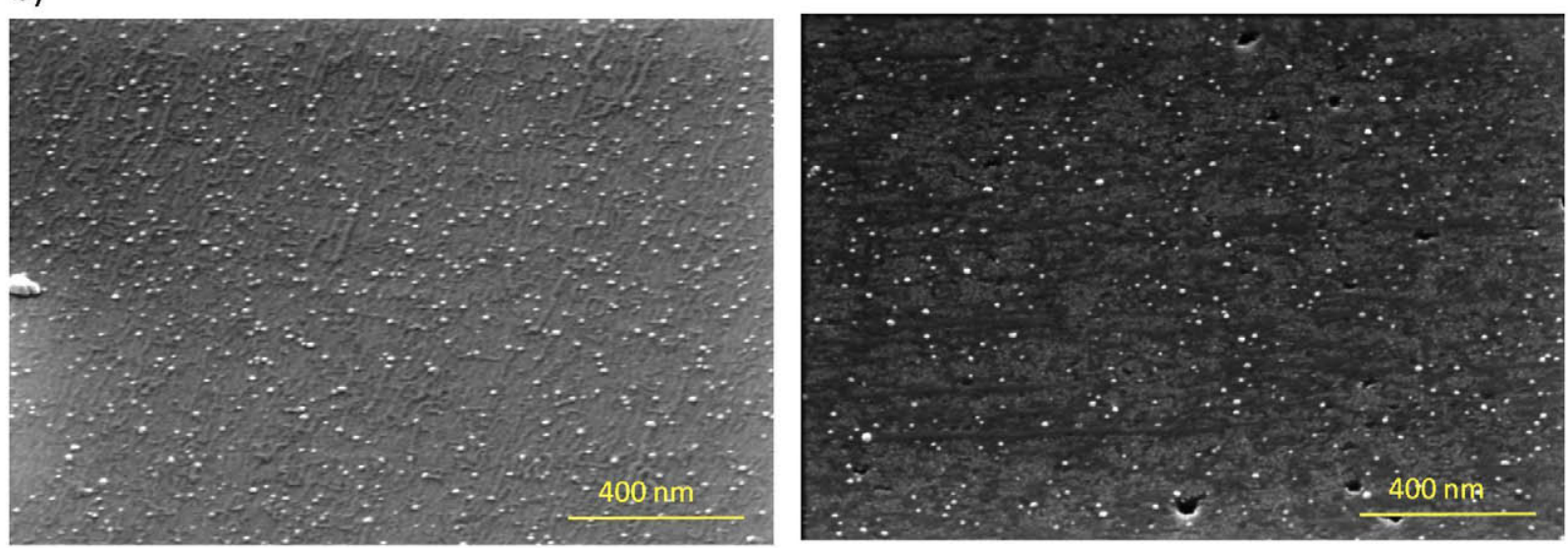

Fig. 5. SEM images of investigated samples: a) NI52n as grown (left) and after RTA process (right); b) NI74n as grown (left) and after RTA process (right).

a)

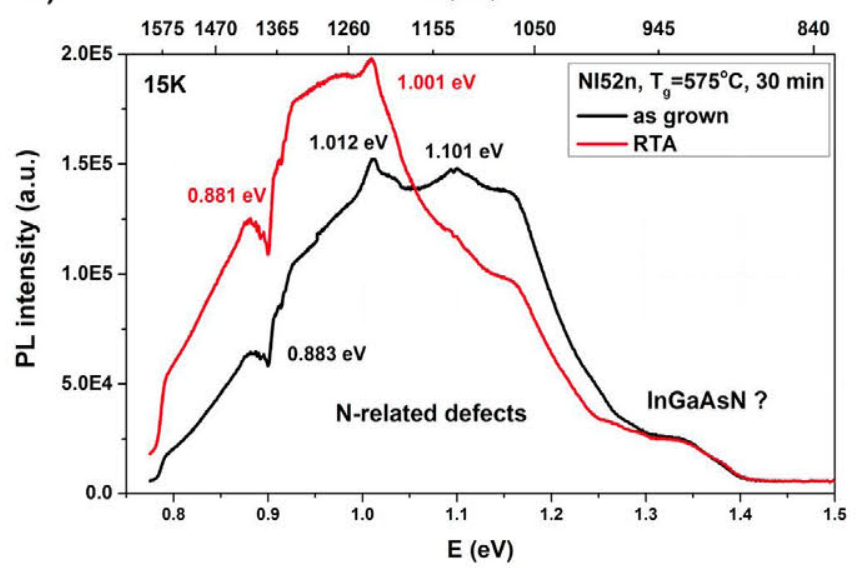

b)

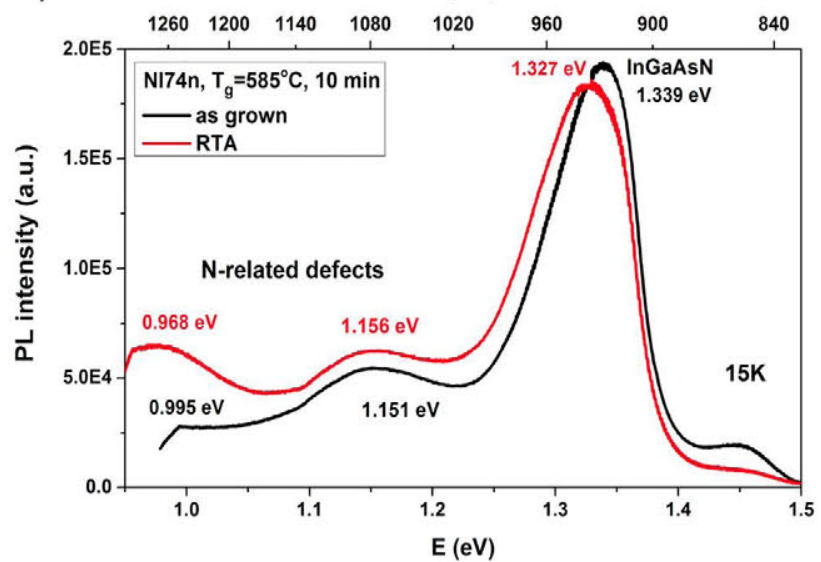

Fig. 6. Low temperature PL spectra of investigated samples: a) NI52n as grown and after RTA process; b) NI74n as grown and after RTA process.

The two smaller binding energy contributions (396.8 and $398.9 \mathrm{eV}$ ) have been described in previous works, and they correspond to $\mathrm{N}-\mathrm{In}$ and N-Ga bonds, respectively [12-14,24-28]. The third contribution origin is not evident. Hydrazines show binding energies in this range $[25,29]$, therefore, precursor debris should be considered as one of the possible origins, more probably $\mathrm{NH}_{2}$ coming from TBHy pyrolysis [13]. Some authors have attributed this binding energy signal to $\mathrm{N}-\mathrm{O}$ bonds [14,30]. However, its increasing intensity with explored depth points to another possible origin in $\mathrm{N}$-As bonds, i.e. $\mathrm{N}$-As dimers in As site [25]. None of the three origins can be discarded and this third contribution would be the sum of them. The fourth component, at the higher binding energy, would be related to $\mathrm{N}-\mathrm{N}$ bonds, i.e. interstitial $\mathrm{N}$ dimer on the As site $(\mathrm{N}-\mathrm{N}$ split interstitial), as it has been previously described by some other authors $[24,28,30]$. Similar core level deconvolutions can be made for the rest of the samples.

Fig. $7 \mathrm{~b}$ represents the evolution of the $\mathrm{N}-\mathrm{In}$ and the $\mathrm{N}-\mathrm{Ga}$ bond contributions with explored depth for the studied samples. For all of them, the N1 s core level signal at the lowest explored depth is dominated by the N-Ga contribution, which is favoured by the chemical 

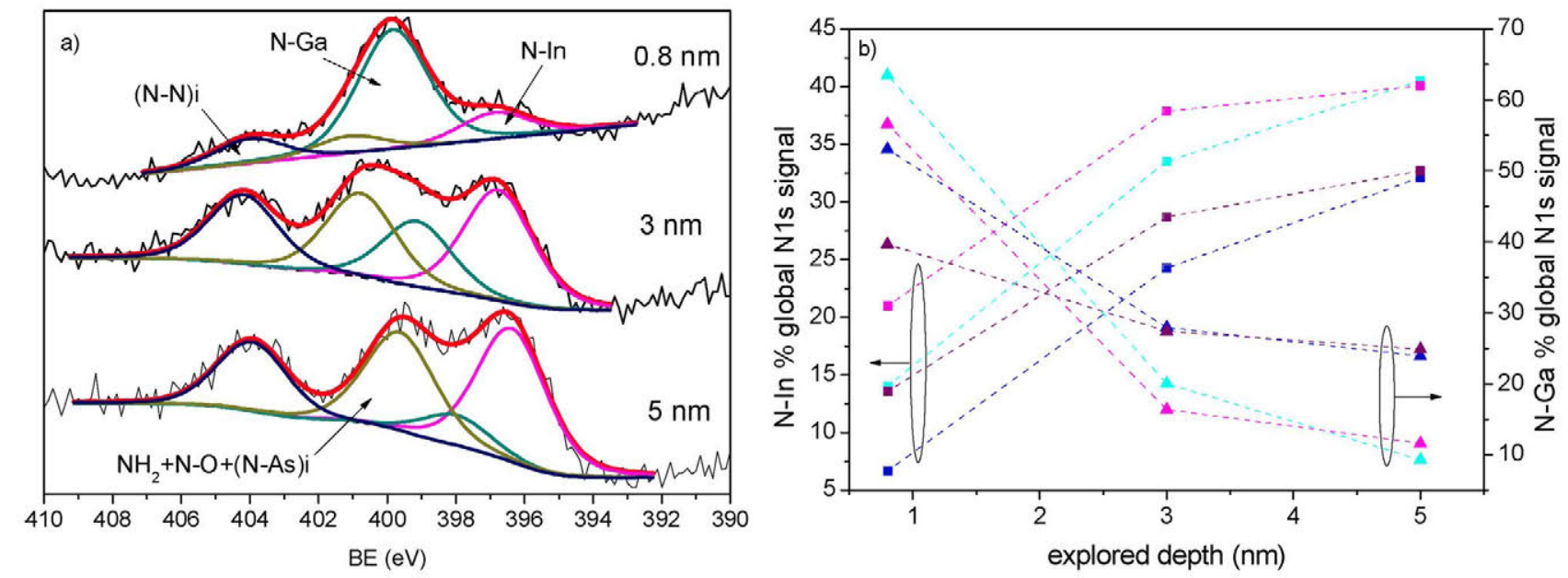

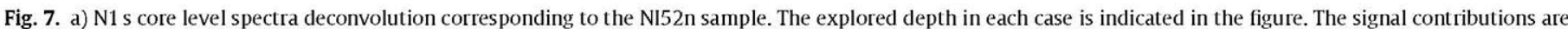

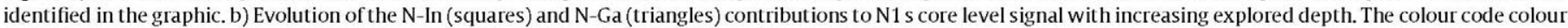
is: NI52n, magenta; NI52 nA, purple; NI74n, cyan; NI74nA, blue. Note the different Y-scales in this graphic.

dynamics during the layer growth, due to the excess Ga at the topmost surface. This fact distorts the intensity distribution among the different contributions at this explored depth (see Fig. 7a) and hinders a clear explanation of each contribution intensity evolution. Higher growth temperature moderates this tendency, probably due to the formation of a considerable amount of $\mathrm{Ga}_{2} \mathrm{O}_{3}$ on the NI74n sample surface (see Ga2 $\mathrm{p}_{3 / 2}$ core level analysis in the SI). RTA treatment also diminishes the N-Ga contribution intensity irrespective of the growth temperature. Again the formation of $\mathrm{Ga}_{2} \mathrm{O}_{3}$ at the topmost surface due to the temperature reached during annealing could be the explanation of this fact [31], as it is confirmed by the analysis of the $\mathrm{Ga}_{2} \mathrm{p}_{3 / 2}$ core level signal (see SI file). As explored depth increases, the N-In amount increases while N-Ga amount decreases. When comparing both as-grown samples, the ratio [NIn]/[N-Ga] is always bigger for NI74n sample, except at the biggest explored depth, where it reaches a similar value for both samples, $\sim 4$. The reason for that could be the diminution of In content as explored depth increases, according to HRXRD results (Fig. 2). This diminution is more abrupt for the NI74n sample, since in $20 \mathrm{~nm}$, In amount decreases to nearly half of the value at surface. It means that, assuming a linear decrease along the layer thickness, considering the first four nanometers from the top surface, in the NI74n sample, the In amount decreases $20 \%$ with respect to the value at the surface, while for NI52n sample, it only decreases $5 \%$. Therefore, in spite of the same figure obtained for the ratio [N-In]/[N-Ga], the proportion of In cations linked to $\mathrm{N}$ would be higher for the sample grown at $585^{\circ} \mathrm{C}, \mathrm{N} 174 \mathrm{n}$. After RTA treatment, the amount of $\mathrm{N}$-Ga bonds is higher than in the as-grown samples for the explored depths 3 and $5 \mathrm{~nm}$. This is probably due to the As evaporation during thermal annealing, which releases Ga cations, that would tend to get bonded to excess $\mathrm{N}$ in the sample surface. Consequently, $\mathrm{N}$-In bonds amount is smaller for the annealed samples, irrespective of the growth temperature.

The behaviour of the other two contributions to this core level as explored depth increases and the influence of growth temperature and RTA treatment on their intensities, have been resumed in Figs. $8 \mathrm{a}$ and $8 \mathrm{~b}$. First one concerns to the triple origin contribution $\mathrm{NH}_{2}+\mathrm{N}-\mathrm{O}+\mathrm{N}-$ As. Both, the precursor debris $\left(\mathrm{NH}_{2}\right)$, as well as $\mathrm{N}-\mathrm{O}$ components, should diminish as explored depth increases, therefore, the increasing intensity of this contribution with explored depth for the as-grown samples can only be due to the N-As dimers. The higher intensity of the third contribution for the NI74n sample, as compared to NI52n (see Fig. 8a), could be related with the higher precursor debris amount for the first sample as it is demon- strated for some other core levels (see SI file). The RTA treatment seems to have different effect depending on the explored depth. It is remarkable the fact that for both annealed samples, the intensity of the $\mathrm{NH}_{2}+\mathrm{N}-\mathrm{O}+\mathrm{N}$-As contribution is rather higher than for the as-grown samples at the topmost surface (explored depth $0.8 \mathrm{~nm}$ ). However, the contribution intensity decreases as explored depth increases for the annealed samples. N-As bonds formation may get enhanced at the topmost surface due to the combination of two factors: As evaporation during RTA, and the N excess at the topmost surface due to the RTA carried out in $\mathrm{N}_{2}$ atmosphere, that may induce the formation of these defects, just on top of the annealed samples. Regarding the highest binding energy contribution at $404.4 \mathrm{eV}$, its origin is attributed to interstitial dimers $\mathrm{N}-\mathrm{N}$, one of the most common $\mathrm{N}$-related defects in these compounds [11]. A higher growth temperature clearly hinders the formation of such kind of defects (Fig. 8b). Conversely, RTA treatment seems to favour its presence, especially for the samples grown at higher temperature, where a remarkable increase of this contribution has been detected for the annealed sample (NI74 nA) as compared to the as-grown one (NI74n) (Fig. 8b). It seems that RTA process in the case of samples grown at higher temperature will more efficiently form $(\mathrm{N}-\mathrm{N})_{i}$ better than $\mathrm{N}-$ As defects.

\subsubsection{In $3 d$ core level}

For this core level, there are three contributions at every explored depth, as it is seen in Fig. 9. The data analysis has been made taken into account the satellite peaks appearing in the $3 \mathrm{~d}_{5 / 2}$ doublet constituent due to the non-monochromaticity of the $\mathrm{Mg}-\mathrm{K}_{\alpha}$ anode. The two most intense contributions correspond to bonds In-As (lower binding energy) and In-O (intermediate binding energy). These contribution origins are rather clear and their binding energies match the literature results [29,32 and references therein]. They both exhibit the same evolution with explored depth for all the samples. In-As contribution increases while In-O contribution decreases as explored depth increases (Figure 1010a). The third contribution is the one at the highest binding energy. It decreases as the explored depth increases and this fact suggests the precursor debris as one of its possible origins. However, the analysis of this core level in an InGaAs sample showed that at the take-off angle $90^{\circ}$ ( $5 \mathrm{~nm}$ explored depth), the contribution from precursor debris was negligible. Therefore, at this take-off angle, the small contribution seen on the left side of the signal for both doublet components should be assigned to In-N bonds [33]. 

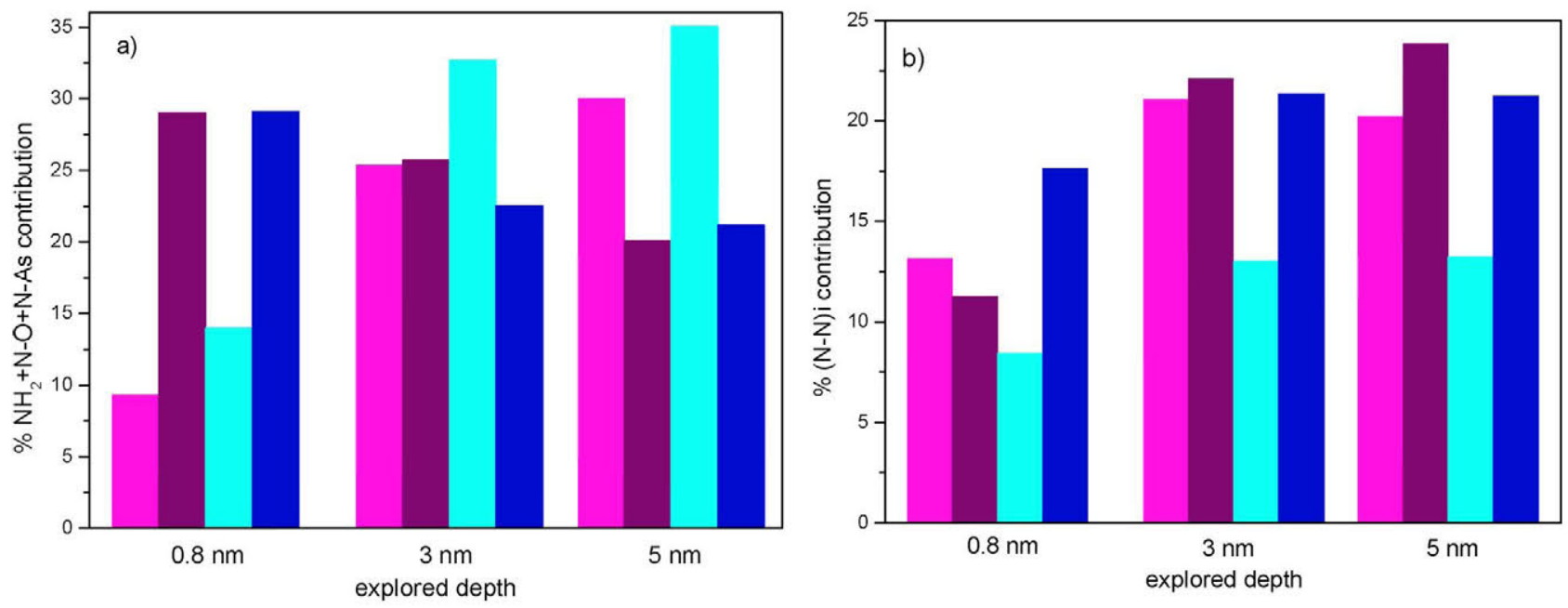

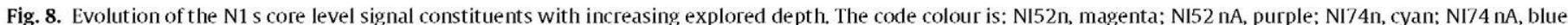
a) $\mathrm{NH}_{2}+\mathrm{N}-\mathrm{O}+\mathrm{N}$-As and b) $(\mathrm{N}-\mathrm{N})_{i}$ contributions.

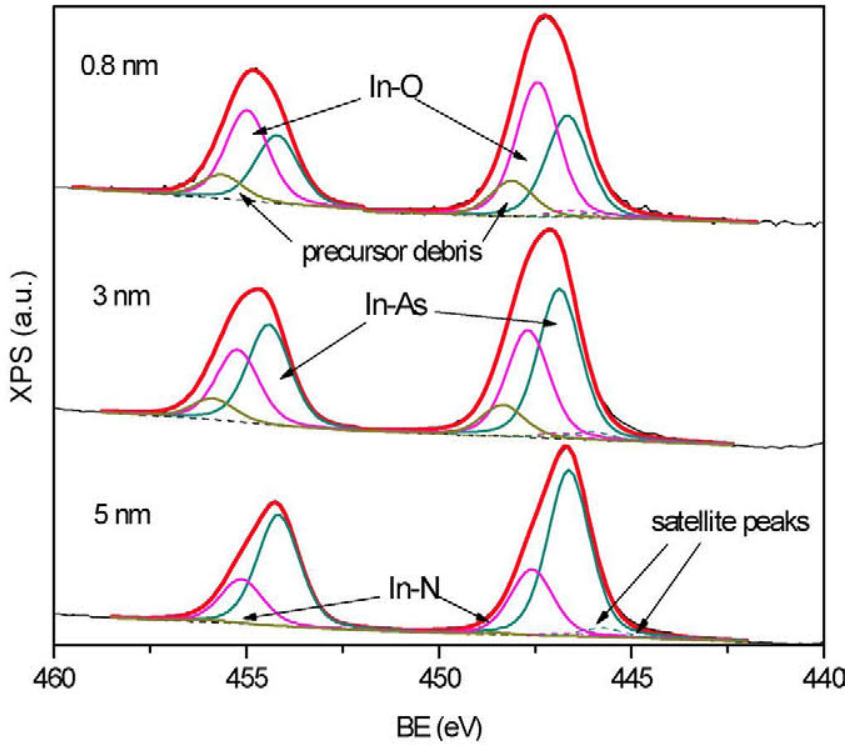

Fig. 9. In $3 d$ core level spectrum deconvolution corresponding to the NI74n sample. The explored depth in each case is indicated in the figure. The signal contributions are identified in the graphic.
The quantity of In oxide is a bit smaller for the as-grown sample prepared at lower temperature. When comparing samples before and after the RTA treatment, the first conclusion is that annealing removes part of the In oxide trapped on the sample surface. Therefore, the lowest energy contribution always raises up after annealing, due to the formation of new In-As bonds. This effect is much more pronounced for the samples prepared at higher temperature (Fig. 10a).

Regarding the influence of the temperature on the formation of In-N bonds, when comparing [In-N]/[In-As] ratio for both as-grown samples, the figures obtained, $1.23 \%$ (NI52n) and 1.33\% (NI74n), confirm what was concluded from $\mathrm{N} 1 \mathrm{~s}$ core level analysis, the higher growth temperature, the more probable formation of In-N bonds in these samples. Fig. 10b illustrates this fact, showing the contribution of the In-N bonds to the In $3 \mathrm{~d}$ core level global intensity for the data reaching $5 \mathrm{~nm}$ explored depth. Li and coworkers showed an opposite tendency with temperature, although in this case, samples were grown by MBE at much lower growth temperature [33]. RTA treatment seems to affect this contribution in a different way depending on the sample growth temperature. There is a clear intensity diminution for the samples grown at low temperature (NI52 nA), while, on the contrary, a small intensity increase is detected for the samples grown at high temperature (NI74 nA)
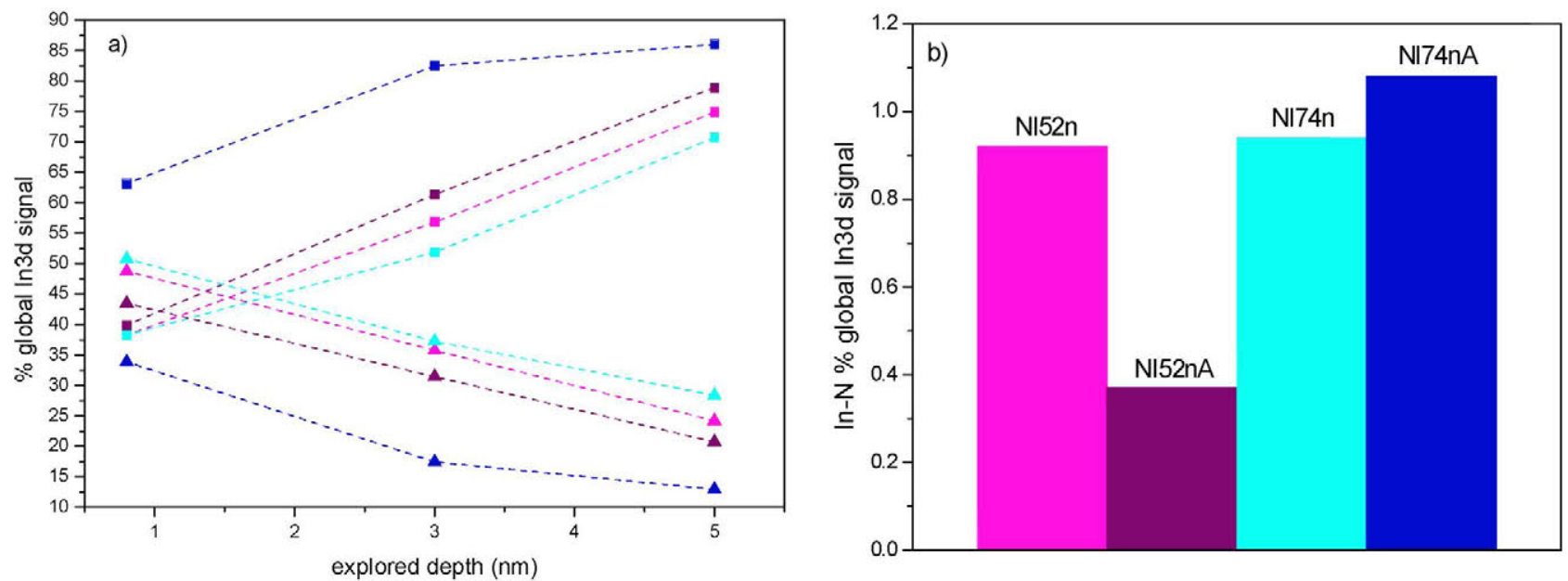

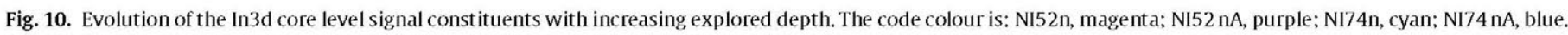
a) In-As (squares) and In- $\mathrm{O}$ (triangles) contributions. b) In- $\mathrm{N}$ contribution. Data correspond to $5 \mathrm{~nm}$ explored depth. 
(Fig. 10b). However, in this last case, it is worth remaining here the remarkable decrease of the In-O contribution after annealing, much more important than for NI52 nA samples (Fig. 10a). That implies that the [In-N]/[In-As] ratio is always smaller for the annealed samples when compared to the as-grown ones (0.5\% for NI52 nA and $1.25 \%$ for NI74 nA). Therefore, this RTA process will rather promote the formation of In-As instead of N-In bonds [32], as it was also concluded from the analysis of the N1 s core level. A careful revision of the RTA temperature and time is compulsory in order to achieve the expected results in terms of In-N bonds enhancement.

\section{Conclusions}

The influence of growth temperature and post growth annealing on the $\mathrm{N}$ coordination chemistry of InGaAsN layers has been studied using XPS and some other characterisation techniques. The amount of precursor debris and different oxides on the sample surfaces has additionally been correlated with the growing conditions. For such purpose, strained and relaxed InGaAsN layers have been grown using AP-MOVPE. The lattice strain is more dependent on the growth conditions (temperature and time) rather than on the postgrowth annealing and the growth temperature is also the main responsible of the surface roughness. Thicker samples prepared at lower temperature are relaxed and misfit dislocations appear at their surfaces, which look with the typical crosshatch morphology. On the contrary, thinner samples prepared at higher temperature are strained, though the RTA treatment reduces partially the strain by increasing the lattice relaxation. Surface roughness is related to the presence of misfit dislocations, but also to the presence of precursor debris observed for the as-grown samples. The annealing process induces As evaporation and therefore the formation of voids at the sample surfaces as well as it enhances the formation of new Ga-N bonds for the higher explored depths, due to the decrease of As-N defects. A higher growth temperature clearly enhances the formation of $\mathrm{N}$-In bonds, though accompanied by a more important presence of precursor debris. Their amount can be decreased by post-growth annealing, however the amount of $(\mathrm{N}-\mathrm{N})_{i}$ split interstitial defects increases during this process.

The presented studies clearly show that the surface chemistry analysis by means of XPS supported by other examination methods help to understand the processes responsible for the material quality of as grown and annealed InGaAsN epilayers. The obtained results indicate that the growth temperature as well as the post growth annealing conditions have to be carefully optimized in order to improve the structural and optical properties of InGaAsN alloys. The analysis connected with almost fully relaxed epilayers (NI52n and NI52 nA samples) are especially important due to a lack of literature data.

\section{Acknowledgments}

This work was co-financed by Wroclaw University of Science and Technology statutory grants and by the MINECO (Spain) through projects TEC2014-54260-C3-3-P and PCIN2015-0181C02-01. Authors would like to thank K. Żelazna, MSc., A. Szyszka, Ph.D., and J. Serafińczuk, Ph.D., from Wrocław University of Science and Technology for the PL, AFM and HRXRD measurements, and the SCBI and SCAI staff at the University of Málaga.

\section{Appendix A. Supplementary data}

Supplementary data associated with this article can be found, in the online version, at https://doi.org/10.1016/j.apsusc.2017.10. 032.

\section{References}

[1] M. Weyers, M. Sato, H. Ando, Jpn. J. Appl, Phys. 31 (1992) L853-L855.

[2] M. Kondow, K. Uomi, A. Niwa, T. Kitatani, S. Watahiki, Y. Yazawa, Jpn. J. Appl, Phys. 35 (1996) 1273-1275.

[3] U. Tisch, E. Finkman, J. Salzman, Appl. Phys. Lett. 81 (2002) 463-465.

[4] K.M. Kim, J.-I. Lee, J.H. Ryu, J. Ceramic Proc. Res. 16 (2015) 45-48.

[5] M. Henini, Dilute Nitride Semiconductors, 1st edition, Elsevier Science, 2004.

[6] A. Erol, Dilute III-V Nitride Semiconductors and Material Systems: Physics and Technology, Springer, 2010.

[7] D. Gollub, S. Moses, M. Fischer, A. Forchel, Electronics Lett. 39 (2003) 777-778

[8] H.-P.D. Yang, C. Lu, R. Hsiao, C. Chiou, C. Lee, C. Huang, H. Yu, C. Wang, K. Lin, N.A. Maleev, A.R. Kovsh, C. Sung, C. Lai, J. Wang, J. Chen, T. Lee, J.Y. Chi, Semicond. Sci. Technol. 20 (2005) 834-839.

[9] V. Sabnis, H. Yuen, M. Wiemer, AIP Conf. Proc, 1477 (2012) 14-19.

[10] A. Kosa, L. Stuchlikova, L. Harmatha, M. Mikolasek, J. Kováč, B. Ściana, W. Dawidowski, D. Radziewicz, M. Tłaczała, Sol. Energy 132 (2016) 587.

[11] K. Laaksonen, H.-P. Komsa, T.T. Rantala, R.M. Nieminen, J. Phys.: Condens, Matter 20 (2008) 235231

[12] W. Chang, J. Lin, W. Zhou, S.J. Chua, Z.C. Feng, Appl. Phys. Lett. 79 (2001) 4497-4499.

[13] T.D. Veal, I. Mahboob, L.F.J. Piper, T. Ashley, M. Hopkinson, C.F. McConville, J. Phys.: Condens. Matter 16(2004) S3201-S3214.

[14] T.S. Lay, W.T. Kuo, L.P. Chen, Y.H. Lai, W.H. Hung, J.S. Wang, J.Y. Chi, D.K. Shih, H.H. Lin, J. Vac. Sci. Technol. B 22 (2004) 1491-1494.

[15] B. Ściana, D. Radziewicz, D. Pucicki, J. Serafińczuk, W. Dawidowski, K. Bielak, M. Badura, Gelczuk Ł, M. Tłaczała, M. Latkowska, P. Kamyczek, J. Kováč, M. Florovič, Andrej Vincze, Proc. SPIE 8902 (2013) 89020J.

[16] B. Ściana, D. Radziewicz, D. Pucicki, I. Zborowska-Lindert, J. Serafińczuk, M. Tłaczała, M. Latkowska, J. Kováč, R. Srnanek, Cryst, Res. Technol. 47 (2012) $313-320$.

[17] W. Dawidowski, B. Ściana, M. Latkowska, D. Radziewicz, D. Pucicki, K. Bielak, M. Badura, M. Tłaczała, Proc, SPIE 8902 (2013) 89022G

[18] XPSPeak 4.1, http://xpspeak.software.informer.com/4.1/.

[19] D. Pucicki, K. Bielak, B. Sciana, W. Dawidowski, K. Zelazna, J. Serafińczuk, J. Kováč, A. Vincze, Ł. Gelczuk, P. Dluzéwski, J. Elect. Eng. 65 (2014) 299-2303.

[20] D. Pucicki, K. Bielak, B. Ściana, D. Radziewicz, M. Latkowska-Baranowska, ]. Kovác, A. Vincze, M. Tłacz ała, J. Cryst. Growth 433 (2016) 105-113.

[21] A. Albo, C. Cytermann, G. Bahir, D. Fekete, Appl.Phys. Lett. 96 (2010) 141102

[22] R. Kudrawiec, Photoreflectance and Photoluminescence Investigations of Nitrogen Diluted III-V Alloys and Their Low Dimensional Structures, PhD Thesis, Wroclaw University of Technology, Poland, 2004.

[23] K.M. Kim, W.-B. Kim, D. Krishnamurthy, J.H. Ryu, S. Hasegawa, H. Asahi, J. Cryst. Growth 368 (2013) 35-38.

[24] J.-D. Hecht, F. Frost, D. Hirsch, H. Neumann, A. Schindler, A.B. Preobrajenski, T. Chassé, J. Appl. Phys. 90 (2001) 6066-6069.

[25] V.L. Berkovits, V.P. Ulin, M. Losurdo, P. Capezzuto, G. Bruno, G. Perna, V. Capozzi, Appl. Phys. Lett. 80 (2002) 3739-3741.

[26] T. Hoshii, M. Yokoyama, H. Yamada, M. Hata, T. Yasuda, M. Takenaka, S. Takagi, Appl. Phys, Lett, 97 (2010) 132102.

[27] F. Ishikawa, S. Fuyuno, K. Higashi, M. Kondow, M. Machida, H. Oji, J.Y. Son, A. Trampert, K. Umeno, Y. Furukawa, A. Wakahara, Appl. Phys, Lett. 98 (2011) 121915.

[28] X.Z. Chen, D.H. Zhang, Y.]. Jin, J.H. Li, J.H. Teng, N. Yakovlev, J. Cryst. Growth 362 (2013) 197-201

[29] Handbook of X-ray Photoelectron Spectroscopy; Moulder, Stickle, Sobol, Bomben, Ed. Physical Electronics Inc., 1995.

[30] Y. Wang, D.H. Zhang, X.Z. Chen, Y.J. Jin, J.H. Li, C.J. Liu, A.T.S. Wee, S. Zhang, A. Ramam, Appl. Phys. Lett. 101 (2012) 021905.

[31] Y. Jeliazova, R. Franchy, Surf. Sci. 527 (2003) 57-70.

[32] M.C. López-Escalante, M. Gabás, I. García, E. Barrigón, I. Rey-Stolle, C. Algora, S. Palanco, J.R. Ramos-Barrado, Appl. Surf. Sci. 360 (2016) 477-484.

[33] J. Li, S. Zhang, F. Gao, L. Wen, S. Zhou, G. Li, J. Appl. Phys, 117 (2015) 055304, 